\title{
Short-term spatial memory in honeybees
}

\author{
M. R. ISNEC, P. A. COUVILLON, and M. E. BITTERMAN \\ University of Hawaii, Honolulu, Hawaii
}

\begin{abstract}
Foraging honeybees were trained individually in two-choice spatial problems. Differentially rewarded for spatial alternation in Experiment 1 ("win-shift" training), they showed instead a clear tendency to perseverate- that is, to prefer on each trial the location of reward on the immediately preceding trial. On the basis of the results of Experiments 2 and 3, in which one location was rewarded over shorter or longer series of consecutive trials, an associative interpretation of the perseveration found in the first experiment was rejected in favor of an interpretation in terms of short-term spatial memory. Experiment 4, in which the animals were rewarded on each trial for choosing either location, also showed perseveration. Honeybees, like rats, seem to remember a rewarded location recently visited, but tend to return to it rather than, like rats, to avoid it.
\end{abstract}

In recent experiments by Brown and Demas (1994; see also Demas \& Brown, 1995), honeybees were studied in an analogue of the radial maze designed by Olton and Samuelson (1976) for the study of short-term memory in rats. Confronted on each trial with an array of six baited targets, the honeybees showed some tendency to avoid targets visited earlier on the trial, from which Brown and Demas concluded that the control of performance by short-term or "working" memory, as known in a variety of vertebrate species, is to be found also in honeybees. The conclusion is difficult, however, to accept in the light of a series of criticisms of the technique employed by Brown and Demas that have been offered by Burmeister, Couvillon, and Bitterman (1995). In subsequent experiments not open to those criticisms, Burmeister et al. used arrays of three or four targets and found only some rather marked position biases that might conceivably have masked any short-term memory effects. They suggested that subsequent work be done in even simpler situations, analogues of the two-choice situations in which the reluctance of rats to return to recently visited locations was first clearly established (see, e.g., Glanzer, 1953; Montgomery, 1952).

Ohyama, Couvillon, and Bitterman (1995) trained individual foragers with targets of two different colors, rewarding them on each trial for choosing the nonrewarded color of the immediately preceding trial (alternation or "win-shift" training). The animals did not learn to alternate, but from the very beginning showed instead a reliable tendency to perseverate in the choice of color-that is, to choose on each trial the rewarded color of the im-

This work was supported in part by Grant IBN-9308132 from the National Science Foundation and in part by Research Centers for Minority Institutions Grant RR03061 from the National Institutes of Health. The participation of M. R. Isnec was made possible by Minority Biomedical Research Support Grant GM08125-22 from the National Institutes of Health. Correspondence should be addressed to M. E. Bitterman, Békésy Laboratory of Neurobiology, 1993 East-West Road, Honolulu, HI 96822 (e-mail: jeffb@pbrc.hawaii.edu). mediately preceding trial. There was perseveration also in the choice of location. The colored targets between which the animals were permitted to choose on each trial were presented at two fixed locations, each color as often in each location as in the other and as likely to be rewarded in each location as in the other. Although not differentially rewarded either for spatial alternation or for spatial perseveration, the animals showed - again from the very beginning - a reliable tendency to go on each trial to the location of reward on the immediately preceding trial.

As noted by Ohyama et al. (1995), the interpretation of the color effect is complicated because the training procedure - adapted from Waddington and Gottlieb (1990) was such that each choice was made in the presence of the most recently rewarded color. After an animal had found the correct target on any trial and was ingesting the small drop of sucrose given as reward, the target with the animal feeding in it was moved to the choice point, and two fresh targets were introduced at the old locations. From there, after finishing the sucrose, the animal would fly up, hover over the situation, and make its next choice. The possibility must therefore be considered that the perseveration in the choice of color was influenced in some way by the continued presence of the previously rewarded color as distinct from short-term memory. With the choice point away from the previously rewarded location and equidistant from the two alternatives, perseveration in the choice of location is, by contrast, more likely to have been an unbiased mnemonic effect of some kind.

How is the spatial perseveration to be explained? The simplest possibility is that it can be understood entirely in associative terms-on the assumption that the attractiveness of a location is a function of the strength of its association with reward, which is increased by each reward and decreased by each nonreward in the same way here as in conventional training with choice of a given location consistently rewarded (e.g., Huber, Couvillon, \& Bitterman, 1994). Another possibility is that short-term memory plays a role, serving in honeybees to increase (rather than, as in rats, to decrease) the attractiveness of 
a recently rewarded location. The assumption that associative learning may in some animals be complemented by transient attractiveness based on short-term memory finds support in an experiment with pigeons by Bailey and Mazur (1990). Choosing between two keys to which responses were rewarded with different probabilities, the pigeons showed both a short-term and a longer term effect of reward; the first was an increased likelihood of choosing the same key on one or two trials immediately following reward for a response to it ("positive recency"), and the second was a much more gradual increase in preference for the key with the higher probability of reward. Our purpose in the present experiments was to look further for evidence of the control of performance by short-term spatial memory in honeybees.

\section{EXPERIMENT 1}

In the experiment by Ohyama et al. (1995) with targets differing in color, color alternation was differentially rewarded and spatial choices were nondifferentially rewarded. In the present experiment, with targets always of the same color, spatial alternation was differentially rewarded. Even though the animals seemed predisposed to perseverate in the choice of location, the possibility seemed worth investigating that they could be trained to alternate, which would provide direct evidence of control by short-term spatial memory. As Dennis (1939) early recognized, to avoid the more recently visited of two locations - for whatever reason-an animal must in some sense remember which it was.

\section{Method}

Subjects. The subjects were 8 foraging honeybees (Apis mellifera), all experimentally naive, from our own hives situated near the laboratory. In this and in the following experiments, the animals were trained individually and sequentially, each in a single experimental session that was several hours in duration.

Apparatus and Procedure. The training situation used in this and in the following experiments is diagrammed in Figure 1. It was a resined plywood enclosure, $58 \mathrm{~cm}$ wide, $58 \mathrm{~cm}$ high, and $46 \mathrm{~cm}$ deep, set into a laboratory window. The enclosure was open to the outside, and it was fitted with a pair of sliding Plexiglas panels through which the observer had access to the enclosure from the inside. The targets used were plastic cones like that sketched in Figure 2; they were $6.2 \mathrm{~cm}$ at the widest diameter, $2 \mathrm{~cm}$ high, and $28 \mathrm{ml}$ in volume. Their inside surfaces were blue. The center-to-center distance from locations $A$ to $B$ and from $A$ to $C$ was $42 \mathrm{~cm}$; that from $B$ to C, $48 \mathrm{~cm}$.

Each subject was captured in a match box on its arrival at a feeding station providing $10 \%-15 \%$ sucrose solution, carried to the laboratory, and set down at a large drop of $50 \%$ sucrose solution in a single target at location A of the diagram. The subject was marked with a spot of colored lacquer as it fed to repletion, after which it would leave for the hive to deposit the sucrose. The animal normally would come back to the laboratory a few minutes later, continuing thereafter to shuttle between the hive and the training situation as long as sucrose was provided there. If the animal did not return after its first placement, it was carried again to the training situation from the feeding station, where it usually could be found. When the animal did return of its own accord, there was another baited target at location A from which it fed to repletion. The targets used on these pretraining visits and on the subsequent training visits

\section{EXPERIMENTER \\ (INSIDE LABORATORY)}

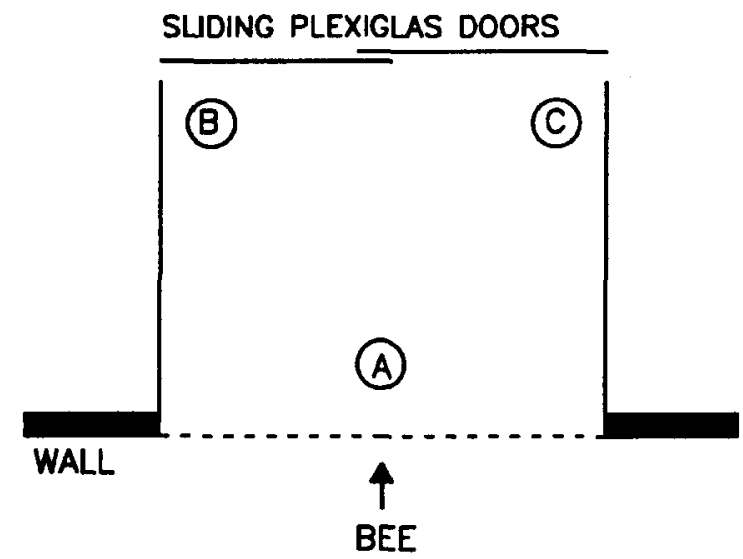

(APPROACHING FROM HIVE)

Figure 1. Diagram of the experimental situation showing the locations $(A, B$, and $C)$ at which targets were placed during training. The enclosure $(58 \mathrm{~cm}$ wide, $58 \mathrm{~cm}$ high, and $46 \mathrm{~cm}$ deep) was open to the outside, and it was fitted with a pair of sliding Plexiglas panels through which the observer had access from the inside. The center-to-center distances from $A$ to $B$ and from $A$ to $C$ were $42 \mathrm{~cm}$, and from $B$ to $C, 48 \mathrm{~cm}$.

were drawn from a large set of otherwise identical targets to which they were returned after washing with water at the conclusion of the visit; the purpose of the procedure (a standard one in this laboratory) is to randomize extraneous stimuli.

The training of the animals, during which they were rewarded equally often at locations $B$ and $C$, was to choose on each trial the nonrewarded location of the immediately preceding trial; that is, spatial alternation was differentially rewarded. Arriving from the hive on each of 24 training visits, each animal found a single target containing a very small $(10-\mu 1)$ drop of $50 \%$ sucrose solution either at location $\mathrm{B}$ or at location $\mathrm{C}-\mathrm{B}$ on half the visits and $\mathrm{C}$ on the rest, in quasi-random sequence. To reach the sucrose, the animal was obliged to land on the inside surface of the target and walk down to the bottom. Then the target with the animal feeding in it was moved to location A, and two additional targets were introduced at locations $\mathrm{B}$ and $\mathrm{C}$. The geometry of the situation (the structure of the targets together with the orientation of the animal) was such that the feeding animal could not see either the targets being introduced or the carefully constrained movements of the experimenter. After ingesting the sucrose, the animal would fly up, hover for a short time over the targets, and then choose between the new ones, of which the target at the previously rewarded location now contained a $10-\mu 1$ drop of water and the other a 10- $\mu$ l drop of sucrose.

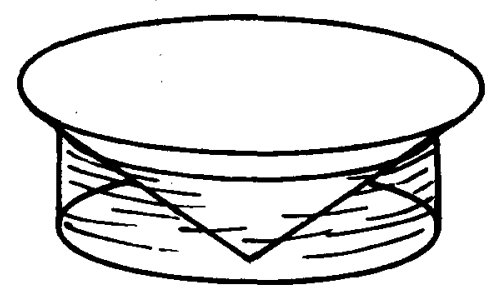

Figure 2. Sketch of a target $(6.2 \mathrm{~cm}$ in its widest diameter, $2 \mathrm{~cm}$ deep, and $28 \mathrm{ml}$ in volume). Each target was a blue plastic cone set in a clear cylindrical base. 
(Water, unacceptable to the animal, could be distinguished from sucrose only by taste; its role was to eliminate the possibility that the correct target could be discriminated on the basis of the presence of the drop of sucrose it contained.) If the animal went first to the target containing water, it was free at once to go to the other, which again with the animal feeding in it was moved to location $A$, and fresh targets were introduced at $B$ and $C$ for the second choice. If the first reward of the visit was at $B$, the second was at $C$, the third was at $B$, and so forth.

The training continued in this way until the animal was replete (that is, after it had taken 50 or $60 \mu \mathrm{l}$ of sucrose) and left of its own accord for the hive to empty its social stomach, returning several minutes later for another series of trials. There were usually four to five choices per visit, the actual number being determined by the animal itself. On each choice trial, the experimenter recorded whether the initial response of the animal was correct or incorrect; incorrect choice was defined as a full landing on the inside surface of the target containing water. It should be emphasized that the use of fresh targets for each choice is critical in such work because of the tendency of foragers to mark depleted flowers with an aversive scent (Giurfa \& Núñez, 1992; Núñez, 1967).

\section{Results and Discussion}

Rewarded on each trial for choosing the nonrewarded location of the preceding trial, the animals in this experiment gave evidence instead of a persistent preference for the rewarded location. Figure 3 shows the mean proportion of incorrect (perseverative) choices in successive blocks of four visits. The proportion of such choices was greater than chance at the outset and did not decline as training continued. The mean overall proportion for the 8 subjects was .60 , which differs significantly from .50 $[t(7)=6.09, p<.01$, two-tailed $]$, and analysis of variance (ANOVA) showed no significant change over blocks of visits $[F(5,35)=1.28, p>.05]$. These results are very much like those of Ohyama et al. (1995), whose targets differed in color as well as in location and whose animals were rewarded for color alternation but nondifferentially rewarded for choice of location.

Although spatial alternation would have constituted strong evidence of control by short-term spatial memory,

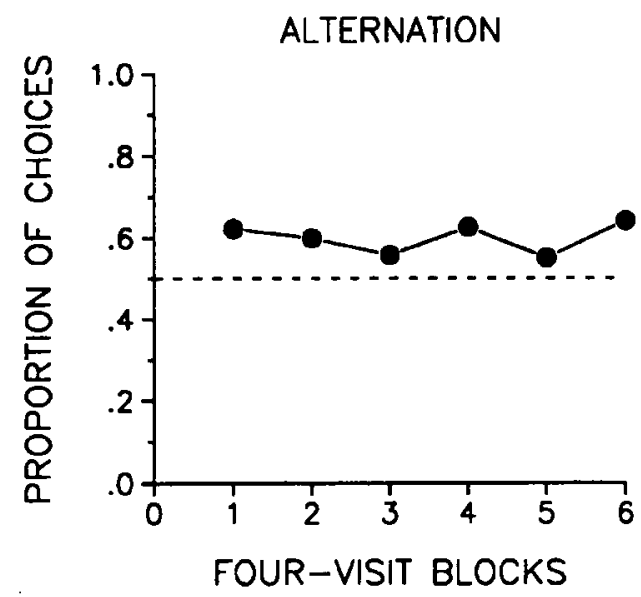

Figure 3. Mean proportion of incorrect (perseverative) choices in successive four-visit blocks of the alternation training in Experiment 1. perseveration is open to explanation in purely associative terms on the assumption that a single trial is sufficient to produce a measurable increase in the strength of association between location and reward. Perhaps the simplest way to decide on the plausibility of the associative explanation of perseveration is to get some information on the cumulative effects of several successive rewards at one of the two locations. A second experiment, carried out under the same general conditions as the first, was intended to provide such information.

\section{EXPERIMENT 2}

In this experiment, the animals were trained as in Experiment 1 , except that the rewarded location was reversed between each visit and the next rather than between each trial and the next (one-visit reversal training). On consecutive trials of any given visit, the rewarded location remained the same. Again, there were usually four to five choices per visit, with the actual number determined by the animal itself. If associative strengthening was responsible for the perseveration found in Experiment 1, the perseveration would be expected here to increase progressively over successive trials within visits.

\section{Method}

Subjects. The subjects were 8 foraging honeybees, all of them experimentally naive, from our own hives.

Procedure. The recruitment and pretraining of the subjects was the same as in Experiment 1 . The training also was the same, except that one of the two locations was rewarded consistently on any given visit. For 4 of the 8 subjects selected at random, B was correct on odd-numbered visits $(1-23)$ and $C$ was correct on evennumbered visits (2-24); for the remaining subjects, the opposite was true.

\section{Results and Discussion}

Figure 4 shows the mean proportion of correct (perseverative) choices on the first four choice trials of each visit pooled over the 24 training visits. (The analysis is not carried beyond the fourth choice trial because there were visits on which some animals made fewer than five choices.) The associative interpretation of the perseveration found in Experiment 1 suggests that in the present experiment the proportion of perseverative choices should have increased from trial to trial within each visit, but it did not. Nor was there any change in performance with continued training, as may be seen in Figure 5, which (like Figure 3, which it closely resembles) is plotted in terms of the mean proportion of correct (perseverative) choices in successive four-visit blocks. An ANOVA showed no significant effect of trials $[F(3,21)<1]$ or of blocks of visits $[F(5,35)<1]$, and no significant trial $\times$ block interaction $[F(15,105)<1]$. The mean overall proportion of perseverative choices was .61 , which differs significantly from $.50[t(7)=4.85, p<.01$, two-tailed $]$. In general, the performance of the one-visit reversal group was almost identical to that of the alternation (one-trial reversal) group in Experiment 1. 


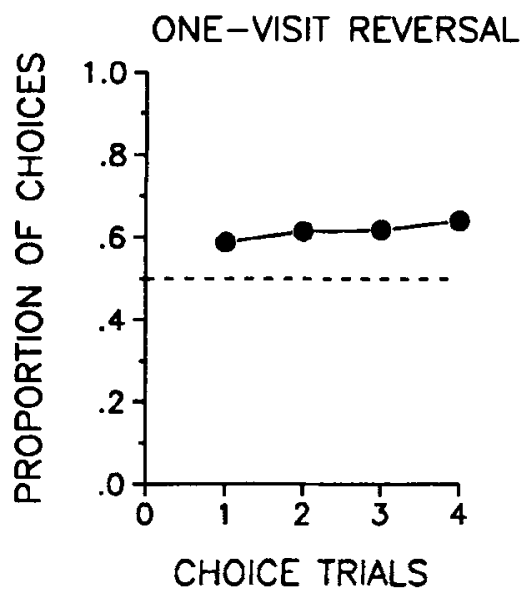

Figure 4. Mean proportion of correct (perseverative) choices on the first four choice trials of each visit pooled over all training visits during the one-visit reversal training of Experiment 2.

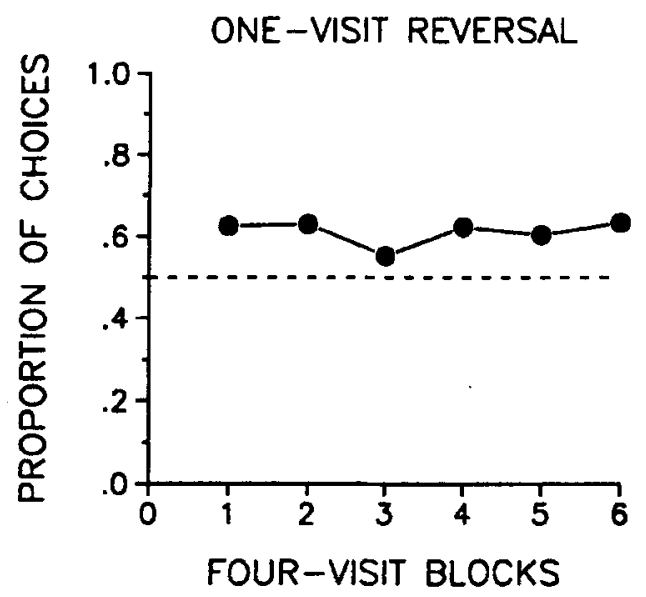

Figure 5. Mean proportion of correct (perseverative) choices in successive four-visit blocks of the one-visit reversal training in Experiment 2.

The fact that as many as four consecutive experiences of reward at a given location proved insufficient to produce a greater preference for that location than was produced by a single reward may raise the question of whether it is possible to demonstrate any associative spatial learning at all under the conditions employed here. The purpose of Experiment 3 was to answer that question.

\section{EXPERIMENT 3}

This experiment was like Experiment 2, but with the positive and negative locations reversed after six training visits rather than after each visit (six-visit reversal training). The increased number of consecutive rewards in each stage of the training was designed to increase the likelihood of an associative perseveration effect.

\begin{abstract}
Method
Subjects. The subjects were 8 foraging honeybees, all of them experimentally naive, from our own hives.

Procedure. The recruitment and pretraining of the subjects were the same as in the previous experiments. The training was like that in Experiment 2, except that here there were only 12 visits, with one location (B for 4 of the 8 subjects selected at random, C for the rest) consistently rewarded on visits 1-6 (approximately 24 trials) and the alternative location on visits 7-12 (approximately 24 trials).
\end{abstract}

\section{Results and Discussion}

Figure 6 shows the mean proportion of correct (perseverative) choices on each of the 12 training visits in Experiment 3. If, in Experiment 2, four consecutive experiences of reward at a given location were not found sufficient to produce a measurable increase in the strength of the preference for that location, the results of the present experiment show that only four more trials (i.e., one additional visit) were required. On the first visit, the performance was like that of the one-visit reversal group in Experiment 2, but by the second visit the proportion of correct choices had risen to more than .80 . On the seventh visit, the cumulative effect of the first six visits was evidenced by a sharp decline in the proportion of correct choices as the animals continued to choose the location rewarded on visits 1-6 rather than the location that now was being rewarded. In subsequent visits, performance steadily improved as (presumably) the association between the new location and reward was gradually strengthened at the expense of the association between the old location and reward. An ANOVA yielded a significant reversal effect, that is, a significant difference in performance on visits $1-6$ as compared with visits $7-12[F(1,7)$ $=13.47, p<.01]$ and a significant effect of visits $[F(5,35)$ $=5.68, p<.01]$, without a significant reversal $\times$ visit interaction $[F(5,35)=1.08, p>.05]$. Clearly there was associative learning about location under the conditions employed here, but associative learning does not provide

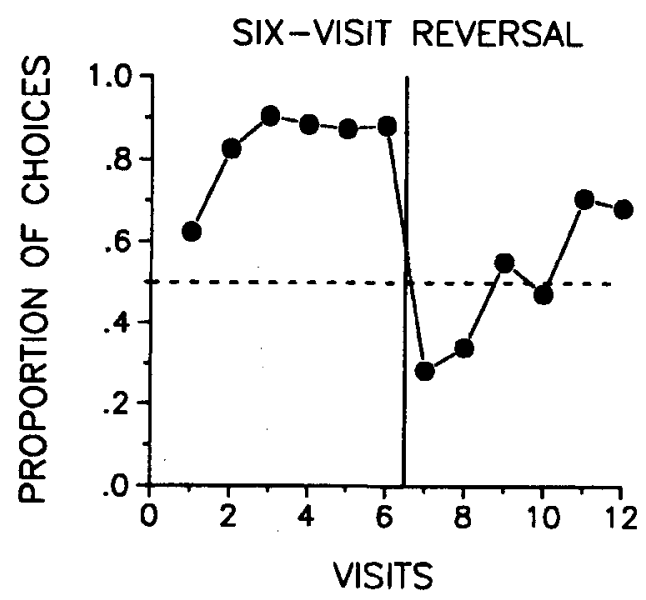

Figure 6. Mean proportion of correct (perseverative) choices on each visit of the six-visit reversal training in Experiment 3. 
a convincing explanation of the perseveration in the choice of location found in the alternation training of Experiment 1 . To account for all of the results in terms of change in associative strength alone, it would be necessary to assume a rather unlikely acquisition function, rising with the first reward, remaining level over the next several rewarded trials, and then rising rapidly again in the course of further rewards.

\section{EXPERIMENT 4}

In a variety of two-choice experiments with rats (e.g., Glanzer, 1953; Heathers, 1940; Montgomery, 1952; Walker, 1956; Wingfield \& Dennis, 1934), spatial alternation was found to be spontaneous in the sense that it was not differentially rewarded. In some cases, the animals were rewarded on each trial for choosing either of the two alternatives; in other cases, neither choice was rewarded. To round out the picture for honeybees, it may be well to examine their performance under such conditions. Since the animals will not work persistently without reward, Experiment 4 was done with reward on each trial for choice of either of the two locations.

\section{Method}

Subjects. The subjects were 8 foraging honeybees, all of them experimentally naive, from our own hives.

Procedure. The recruitment and pretraining of the subjects were the same as in the previous experiments. The training also was the same, except that the choice on each trial was between two targets, both of which contained sucrose. The number of visits was set at 24 .

\section{Results and Discussion}

Rewarded on each trial for choosing either of two locations, the animals in this experiment showed the nowfamiliar tendency to choose the location at which reward was taken on the preceding trial. The mean proportion of perseverative location choices in successive blocks of four visits is plotted in Figure 7. The proportion of such choices

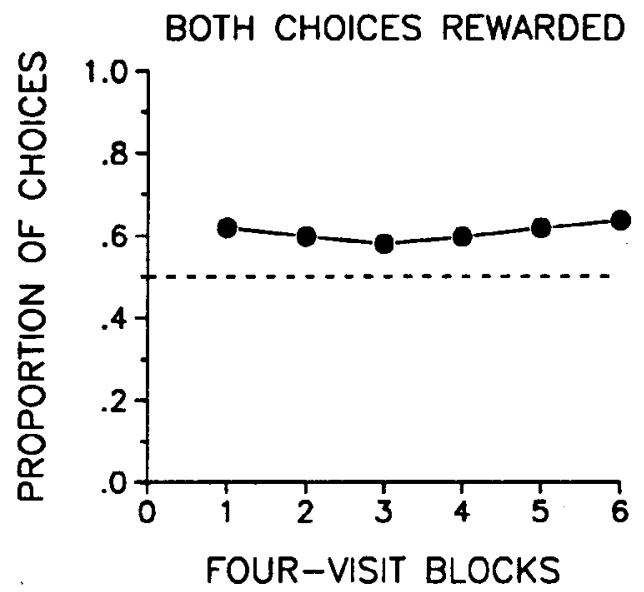

Figure 7. Mean proportion of perseverative choices in successive four-visit blocks of the training in Experiment 4 with choice of either location rewarded. was greater than chance at the outset and did not decline as training continued. The mean overall proportion for the 8 subjects was .61 , which differs significantly from $.50[t(7)=4.21, p<.01$, two-tailed $]$, and an ANOVA gave no indication of significant change over blocks of visits $[F(5,35)<1]$. Evident in the data were some location preferences, modest and fluctuating, that here would have contributed to perseveration and might possibly have masked an alternation tendency; there seems to be no good way to disentangle the two effects. What is clear in any case is that the persistent perseveration is not open to an associative interpretation, according to which the associative strengths of the two alternatives should in the course of consistent reward have approached a common asymptote.

\section{GENERAL DISCUSSION}

If honeybees either alternated spontaneously or could be trained to alternate, control of performance by shortterm spatial memory might readily be inferred (Dennis, 1939). With the honeybees showing perseveration rather than alternation, the inference is much less direct, depending as it does on independent estimates in Experiments 2 and 3 of the (longer term) associative effect of reward, and yet in our opinion the inference from the results of those experiments is reasonable. There is indeed a longer term associative effect, but it develops rather slowly and seems quite unrelated to what can be conceived as an initial perseverative tendency that appears with much the same strength whether the animals are differentially rewarded for alternation (Experiment 1), for perseveration (Experiments 2 and 3), or nondifferentially rewarded (Experiment 4). The plausibility of the assumption that there are two independent processes (short-term memory and associative learning) making for perseveration, which might be questioned on grounds of parsimony, is enhanced by the parallel conclusion drawn from the work with pigeons by Bailey and Mazur (1990) to which reference has already been made.

The failure of our honeybees in Experiment 1 to learn to alternate is noteworthy, given that rats (which tend originally to alternate) can learn very well to perseverate when the location of reward changes unsystematically from trial to trial and they are rewarded for going to the most recently rewarded location (Wolfe \& Spragg, 1934). The failure leads to the suspicion that control by short-term memory may be less extensive in honeybees, whose learning closely resembles the learning of rats in so many other respects (Bitterman, 1988, 1996). As noted, the work of Brown and Demas (1994), who did report avoidance of recently visited places by honeybees, is open to a variety of serious criticisms that have been outlined in some detail by Burmeister et al. (1995, pp. 373-374). A disadvantage of our own method may be that after each reward the feeding animal is moved from the rewarded location to the choice point by the experimenter; there is thus no opportunity for the animal to experience the rewarded lo- 
cation on departure from the target, which in addition to the arrival experience has been found to play an important role in associative spatial learning (Couvillon $\&$ Bitterman, 1992; Gould, 1988).

Much remains to be done in the development of sensitive techniques for the analysis of short-term memory in honeybees. It might be well to try spatial alternation training again, but with consequences of incorrect choice that are more severe than in the present experiments; one way to accomplish that purpose (short of punishment) might be, as the vertebrate literature suggests (e.g., Gonzalez, Bainbridge, \& Bitterman, 1966), to increase the effortfulness of response. Colors might be used in our two-window situation (Lee \& Bitterman, 1990), with the subject shuttling between one window that presents a sample target and an immediately adjacent window that offers a choice between two targets out of sight of the sample. To subvert the perseverative tendency, it might be reasonable to turn to "symbolic" matching (Carter \& Eckerman, 1975), a procedure in which a sample different from either of the alternatives is used to inform the animal's choice.

As noted by Ohyama et al. (1995), the perseveration in the choice of color which they found in honeybees is immediately reminiscent of "flower constancy"-the familiar disposition of individual foragers to confine their visits to flowers of the same plant species (Grant, 1950) and may well contribute to it. If location is narrowly defined as that of a single flower, it is difficult to think of anything like perseveration in the choice of location under field conditions; foraging honeybees tend to move from one flower to the next in a straight line (Waddington, 1980 ), and return to a recently visited location may be discouraged in any case by the presence of the depleted flower, marked with an aversive scent (Giurfa \& Núñez, 1992; Núñez, 1967). Conceivably, however, location should be defined more broadly, and what we are seeing in these experiments is the expression of a tendency on the part of foragers to return to a patch after having left it. Short-term memory of a profitable location that has been newly discovered or rediscovered would be useful if it were stable enough to survive the finder's return to the hive. Under training conditions like those used here, our efforts to find either perseveration or alternation in the choice of location from one visit to the next after only a single large reward on each visit have thus far been unsuccessful.

\section{REFERENCES}

Bailey, J. T., \& Mazur, J. E. (1990). Choice behavior in transition: Development of preference for the higher probability alternative. Journal of the Experimental Analysis of Behavior, 53, 409-422.

BitTERMAN, M. E. (1988). Vertebrate-invertebrate comparisons. In H. J. Jerison \& I. Jerison (Eds.), Intelligence and evolutionary biology (pp. 251-276). Berlin: Springer-Verlag.

BitTERMAN, M. E. (1996). Comparative analysis of learning in honeybees. Animal Learning \& Behavior, 24, 123-141.
Brown, M. F., \& Demas, G. E. (1994). Evidence for spatial working memory in honeybees (Apis mellifera). Journal of Comparative Psychology, 108, 344-352.

Burmeister, S., Couvillon, P. A., \& Bitterman, M. E. (1995). Performance of honeybees in analogues of the rodent radial maze. Animal Learning \& Behavior, 23, 369-375.

Carter, D. E., \& Eckerman, D. A. (1975). Symbolic matching by pigeons: Rate of learning complex discriminations predicted from simple discriminations. Science, 187, 662-664.

Colvillon, P. A., \& BitTerman, M. E. (1992). Landmark learning by honeybees. Journal of Insect Behavior, 5, 123-129.

Demas, G. E., \& Brown, M. F. (1995). Honey bees are predisposed to win-shift but can learn to win-stay. Animal Behaviour, 50, 10411045.

DENNIS, W. (1939). Spontaneous alternation in rats as an indicator of the persistence of stimulus effects. Journal of Comparative Psychology, 28, 305-312.

GiURFA, M., \& NúÑEZ, J. A. (1992). Honeybees mark with scent and reject recently visited flowers. Oecologia, 89, 113-117.

GLANZER, M. (1953). The role of stimulus satiation in spontaneous alternation. Juurnal of Experimental Psychology, 45, 387-393.

Gonzalez, R. C., Bainbridge, P., \& Bitterman, M. E. (1966). Discretetrials lever pressing in the rat as a function of pattern of reinforcement, effortfulness of response, and amount of reward. Journal of Comparative \& Physiological Psychology, 67, 94-105.

Gould, J. L. (1988). Timing of landmark learning by honey bees. Journal of Insect Behavior, 1, 373-378.

Grant, V. (1950). The flower constancy of bees. Botanical Review, 3 , $82-97$.

Heathers, G. L. (1940). The avoidance of repetition of a maze reaction in the rat as a function of the time interval between trials. Journal of Psychology, 10, 259-380.

Huber, B., Couvillon, P. A., \& Bitterman, M. E. (1994). Place and position learning in honeybees (Apis mellffera). Journal of Comparative Psychology, 108, 213-219.

LEE, Y., \& BITTERMAN, M. E. (1990). Learning in honeybees as a function of amount of reward: Control of delay. Animal Learning \& Behavior, 18, 377-386.

Montgomery, K. C. (1952). A test of two explanations of spontaneous alternation. Journal of Comparative \& Physiological Psychology, 45, 287-293.

NúNEZ, J. A. (1967). Sammelbienen markieren versiegte Futterquellen durch Duft. Naturwissenschaften, 54, 322-323.

Ohyama, T., Couvillon, P. A., \& BitTerman, M. E. (1995). Perseveration in the color choices of honeybees. Journal of Insect Behavior, $8,409-415$.

Olton, D. S., \& Samuelson, R. J. (1976). Remembrance of places passed: Spatial memory in rats. Journal of Experimental Psychology: Animal Behavior Processes, 2, 97-116.

WADDINGTON, K. D. (1980). Flight patterns of foraging bees relative to density of artificial flowers and distribution of nectar. Oecologia, 44, 199-294.

Waddington, K. D., \& GottuiEb, N. (1990). Actual vs. perceived probability: A study of floral choice in honeybees. Journal of Insect Behavior, 3, 429-441

WALKER, E. L. (1956). The duration and course of the reaction decrement and the influence of reward. Journal of Comparative \& Physiological Psychology, 49, 167-176.

WINGFIELD, R. C., \& DENNIS, W. (1934). The dependence of the rat's choice of pathways upon the length of the daily trial series. Journal of Comparative Psychology, 18, 135-147.

Wolfe, J. B., \& SPRAGG, S. D. S. (1934). Some experimental tests of "reasoning" in white rats. Journal of Comparative Psychology, 18, 455-469.

(Manuscript received May 1, 1996; revision accepted for publication August 26, 1996.) 